\title{
Clinical Efficacy of Tianmeng Capsule Combined with Paroxetine in Treatment of Menopausal Women with Generalized Anxiety Disorder
}

\author{
Xing'e $\mathrm{Hu}^{1}$, Xiaoxiao $\mathrm{Yu}^{2}$ \\ ${ }^{1}$ Hubei Three Gorges Polytechnic, Yichang, 444300, China \\ ${ }^{2}$ Yichang Central People's Hospital/ Three Gorges University First Clinical Medical College, Yichang, 444300, \\ China
}

Keywords: Generalized anxiety disorder, Tianmeng Capsule, Menopause, Paroxetine, Sex hormones, Oxidative stress

\begin{abstract}
Objective: To study the clinical effect of Tianmeng capsule and paroxetine in the treatment of menopausal women with generalized anxiety disorder. Methods: 63 climacteric patients with generalized anxiety disorder were selected from January 2017 to December 2017. The patients were divided into the control group and the observation group by random number table method, with 63 cases in each group. Patients in the control group were treated with paroxetine, while patients in the observation group were treated with paroxetine and Tianmeng capsule. The difference between the two groups was compared after 1 months of continuous treatment. Results: After treatment, the PSQI and HAMA scores of the two groups were significantly lower than those before treatment $(\mathrm{P}<$ 0.05). After treatment, the PSQI and HAMA scores of the observation group were significantly lower than those of the control group $(\mathrm{P}<0.05)$. The total curative effect rate of the observation group was $93.65 \%$, and that of the control group was $84.13 \%$. The difference between the two groups was statistically significant $(\mathrm{P}<0.05)$. FSH and LH levels after treatment were significantly lower than those before treatment $(\mathrm{P}<0.05)$, and E2 levels were significantly higher than those before treatment $(\mathrm{P}<0.05)$. After treatment, there was no difference in FSH, LH and E2 between the two groups $(\mathrm{P}>0.05)$. After treatment, the levels of SOD and MDA in the two groups were significantly higher than those before treatment $(\mathrm{P}<0.05)$ and lower than those before treatment $(\mathrm{P}<0.05)$. After treatment, the SOD of the observation group was significantly higher than that of the control group $(\mathrm{P}<0.05)$, and MDA was significantly lower than that of the control group $(\mathrm{P}<0.05)$. The rate of adverse reactions in the control group was $25.40 \%$. The rate of adverse reactions in the observation group was $0.00 \%$. There was a significant difference between the two groups $(\mathrm{P}<0.05)$. Conclusion: Paroxetine combined with Sweet Dream Capsule is effective in the treatment of menopausal women with generalized anxiety disorder, with fewer adverse reactions and high safety. It is worthy of clinical promotion.
\end{abstract}

Generalized anxiety disorder (GAD) is a psychiatric disorder, which has a serious negative impact on the life, study, health and normal work of patients. The secretion of sex hormones in menopausal women will be greatly reduced, especially in 45-55 years old menopausal people, about half of them have anxiety and depression symptoms, anxiety disorders. The disease rate also reached the highest in this time period. Menopausal women often suffer from insomnia and tension due to ovarian dysfunction, decreased estrogen levels, and interference from surgery and disease itself. The most commonly used treatment for common menopausal anxious patients is estrogen replacement therapy, giving appropriate amounts of estrogen to the patients. Hormone supplementation can effectively prevent osteoporosis while reducing the mortality of cardiovascular diseases. It can also improve the function of urinary system. However, there are also some drawbacks, to a certain extent, it increases the incidence of breast and cervical cancer. And for generalized anxiety disorder often use serotonin reuptake inhibitors combined with benzodiazepines for treatment, although these two drugs can quickly relieve tension and anxiety, eliminate symptoms, can be more serious and varied adverse 
reactions, patients after treatment prone to myocardial damage, insomnia, constipation, memory disorders, dizziness and dry mouth, Tachycardia, tremor vertigo, blurred vision, orthostatic hypotension and gastrointestinal reactions. The main components of Tianmeng capsule are Lycium barbarum, Codonopsis pilosula, Acanthopanax senticosus and Epimedium, which can regulate the blood-gas balance and improve the endocrine system. In order to explore and analyze the practical effect and application value of Paroxetine Combined with Tianmeng capsule in the treatment of menopausal women with generalized anxiety disorder (GAD), we selected some patients as the observation object to conduct a comparative study. Now we will summarize the reports as follows.

\section{Clinical Material and Methods}

\subsection{Basic Materials.}

In the process of the continuous development and reform of education in China, there are more and more problems in education mode and form. With the continuous development of society, education mode and teaching concept need to adapt to the development and change of the times and constantly innovate and change. Under the background of the Internet + era, we should attach importance to the innovation of the "three dimensional" teaching mode. At the same time, combining the various teaching resources of the network, we can truly achieve the optimal development of the teaching mode under the new educational background. In this regard, this article analyzes and explores the "three dimensional" innovative education mode under the background of Internet plus, and puts forward some specific strategies for the development of "three dimensional" education model.

\subsection{Methods.}

The stereoscopic teaching mode under the background of Internet plus is a new teaching mode developed under the new era environment, so that students can get the coordinated development of teaching process in the whole process of teaching activities, such as basic knowledge cognition, emotional communication and willpower training. The new stereo education mode is the innovation of modern teaching means, and it is also the change of traditional education concept and the reform of traditional education theory. It will play a positive role in promoting and promoting the development of China's overall education mode and future education system.

\subsection{Observation Target.}

First, the implementation of stereo education mode is an inevitable choice for the development of education in the new era of Internet plus education in China. The law of higher education in China stipulates that the teacher's education to the students should conform to the following academic standards: "Students should be made to systematically master the basic theories and basic knowledge necessary for their subject and specialty, master the basic skills, methods and relevant knowledge necessary for their specialty, and have the ability to engage in the actual work and research work of their specialty. In order to realize this standard in the actual education process, teachers must cultivate students' comprehensive learning ability, and must implement the stereo innovative education model. Second, the implementation of stereo education mode is the basic guarantee for the realization of open education in the new era of Internet + education in China. At present, the whole teaching space and teaching thinking mode in the campus are open under the educational environment. In such an educational environment, it is necessary to implement the stereo innovative education mode. Third, the implementation of stereo education mode is an urgent need for quality education in the new era of Internet + education in China. Innovation is the inevitable choice and inevitable road for the development and progress of the times. The stereo innovative education mode is an innovation of educational form and an important measure in educational reform. The core competitiveness of the Internet + era is the ability to innovate. 


\subsection{Statistical Method.}

At the present stage, the realization of innovative education is to match the teaching mode of innovation and the development of the times. Many teachers and leaders agree that the stereo innovative education mode is an important action of teaching innovation. Fourth, the implementation of stereo education mode is the main content of realizing the educational value under the background of Internet + education in the new era. The main functions of modern education include three major functions: teaching, scientific research and social service. To achieve these three educational functions, we must strengthen the implementation of stereo education mode. Fifth, the implementation of stereo education mode is the inevitable trend of China's new era of Internet + education to achieve world education. Education is to serve the country and Society for long-term education, combined with a series of high-quality teaching and academic achievements, as well as the country and society and even the world has contributed to the teaching activities. It can be said that to a certain extent, many countries put the stereo innovative education model in the primary position of the development of education model, and give widespread attention and high attention.

\section{Results}

\subsection{PSQI and HAMA Scores Analysis of the Two Groups before and after Treatment.}

The PSQI and HAMA scores of the two groups were significantly lower than those before treatment $(\mathrm{P}<0.05)$, and the PSQI and HAMA scores of the observation group were significantly lower than those of the control group $(\mathrm{P}<0.05)$. Details are shown in Table 1.

Table 1. PSQI and HAMA scores analysis of the two groups before and after treatment

\begin{tabular}{ccccc}
\hline Group & \multicolumn{2}{c}{ HAMA } & \multicolumn{2}{c}{ PSQI } \\
\hline & Before treatment & After treatment & $\begin{array}{c}\text { Before } \\
\text { treatment }\end{array}$ & After treatment \\
Control group & $35.02 \pm 15.31$ & $9.24 \pm 2.96$ & $9.51 \pm 2.41$ & $9.45 \pm 2.03$ \\
Observation group & $36.91 \pm 13.91$ & $8.15 \pm 2.84$ & $9.91 \pm 2.81$ & $6.59 \pm 1.84$ \\
\hline
\end{tabular}

\subsection{Difference Analysis of Treatment Effect between the Two Groups of Patients.}

The study showed that the total curative effect rate of the observation group was $93.65 \%$, and that of the control group was $84.13 \%$. The difference between the two groups was statistically significant $(\mathrm{P}<0.05)$. Details are shown in Table 2.

Table 2. Difference analysis of treatment effect between the two groups of patients

\begin{tabular}{ccccc}
\hline Group & $\begin{array}{c}\text { marked } \\
\text { effects }\end{array}$ & improvement & Failure & $\begin{array}{c}\text { total effective } \\
\text { rate }\end{array}$ \\
\hline Control group(n=63) & 22 & 31 & 10 & $84.13 \%$ \\
Observation & 30 & 29 & 4 & $93.65 \%$ \\
group(n=63) & & & & 7.153 \\
$\mathrm{X}$ & & & $<0.05$ \\
$\mathrm{P}$ & & & & \\
\hline
\end{tabular}

\subsection{Changes Analysis of Serum Sex Hormones of the Two Groups before and after Treatment.}

FSH and LH levels were significantly lower after treatment in both groups than before treatment $(\mathrm{P}<0.05)$, E2 levels were higher than before treatment $(\mathrm{P}<0.05)$. After treatment, there was no difference in FSH, LH and E2 between the two groups ( $\mathrm{P}>0.05)$. Details are shown in Table 3. 
Table 3. Changes analysis of serum sex hormones of the two groups before and after treatment

\begin{tabular}{ccccccc}
\hline Group & \multicolumn{2}{c}{$\mathrm{FSH}(\mathrm{U} \cdot \mathrm{L})$} & \multicolumn{2}{c}{$\mathrm{LH}(\mathrm{U} \cdot \mathrm{L})$} & \multicolumn{2}{c}{$\mathrm{E}_{2}(\mathrm{ng} \cdot \mathrm{L})$} \\
\cline { 2 - 7 } & Before & After & Before & After & Before & After \\
treatment & treatment & treatment & treatment & treatment & treatment \\
Control group & $60.06 \pm 2.76$ & $54.76 \pm 2.98$ & $49.76 \pm 3.32$ & $46.32 \pm 3.98$ & $47.87 \pm 2.98$ & $49.01 \pm 2.76$ \\
$\begin{array}{c}\text { Observation } \\
\text { group }\end{array}$ & $59.54 \pm 2.43$ & $53.54 \pm 2.32$ & $48.01 \pm 3.87$ & $47.76 \pm 3.65$ & $48.32 \pm 2.43$ & $50.32 \pm 2.98$ \\
\hline
\end{tabular}

\subsection{Difference Analysis of SOD and MDA Levels of the Two Groups before and after} Treatment.

The study showed that the levels of SOD and MDA in the two groups were significantly higher than those before treatment $(\mathrm{P}<0.05)$ and lower than those before treatment $(\mathrm{P}<0.05)$. After treatment, the SOD of the observation group was significantly higher than that of the control group $(\mathrm{P}<0.05)$, and MDA was significantly lower than that of the control group $(\mathrm{P}<0.05)$. Details are shown in Table 4.

Table 4. Difference analysis of SOD and MDA levels of the two groups before and after treatment

\begin{tabular}{ccccc}
\hline Group & \multicolumn{2}{c}{$\operatorname{SOD}(\mu \mathrm{mol} \cdot \mathrm{L})$} & \multicolumn{2}{c}{$\operatorname{MDA}(\mu \mathrm{mol} \cdot \mathrm{L})$} \\
\hline & Before treatment & After treatment & $\begin{array}{c}\text { Before } \\
\text { treatment }\end{array}$ & After treatment \\
Control group & $24.21 \pm 8.76$ & $28.43 \pm 11.21$ & $5.98 \pm 1.43$ & $4.87 \pm 2.01$ \\
Observation group & $25.32 \pm 9.32$ & $33.01 \pm 11.76$ & $5.87 \pm 1.98$ & $4.32 \pm 1.43$ \\
\hline
\end{tabular}

\subsection{Adverse Reactions Analysis of the Two Groups of Patients.}

After treatment, 4 cases of nausea, 7 cases of vomiting, 4 cases of dry mouth and 1 case of blurred vision were found in the control group. The adverse reaction rate was $25.40 \%$. No adverse reactions were observed in the observation group, and the adverse reaction rate was $0.00 \%$. There was a significant difference between the two groups $(\mathrm{P}<0.05)$.

\section{Discussion}

Menopausal generalized anxiety disorder is a common psychiatric disorder in women. Vofloxamine maleate, hydrochloride, tetramethylamine and minoprim hydrochloride are commonly used in clinical treatment. Although they have certain curative effects, there are many adverse reactions, which are not conducive to the rehabilitation of patients. Estrogen is synthesized in the female ovaries and reversibly binds to albumin and sex hormone-binding globulin, and the hormones that do not bind to the outside gradually diffuse to the target tissues and play a special role. Because the main cause of menopausal anxiety is the lack of estrogen secretion, it is often taken to replace the clinical treatment. Recent reports and studies have shown that moderate estrogen administration can improve cognition and promote recovery. The sweet dream capsule used in this study can calm the mind and nourish the heart, strengthen the spleen and stomach, Nourish Qi and kidney, yam epimedium and other traditional Chinese medicine ingredients can also regulate immunity and endocrine, can be very good anti-aging, insomnia treatment, less adverse reactions and side effects, patients will not appear drug dependence and drug withdrawal rebound phenomenon.

The results of this study showed that after treatment, PSQI and HAMA scores of the two groups were significantly decreased compared with those before treatment. Lateral comparison found that the observation group using paroxetine combined with Tianmeng capsule had lower scores than the control group using paroxetine alone, and the total effective rate of treatment was also superior to the former. The difference was statistically significant, fully confirming the actual effect and application 
value of Tianmeng capsule, indicating that the drug can improve the mental state of patients with menopausal anxiety disorders; in addition, we also compared the serum sex hormones, found that there was no significant difference between the two groups before and after treatment, but MDA decreased, SOD has. Therefore, it can be inferred that Tianmeng capsule, unlike ordinary drugs, does not increase hormone levels to protect nerves, but to enhance the ability to play a protective role in antioxidant stress, thereby alleviating symptoms.

Chinese medicine believes that perimenopause is a period when women are extremely weak and tend to decline. The lack of estrogen secretion leads to the loss of protection of related neurons. The body will be stimulated by various emergency, secretory system disorders, and the rise of ROS caused by oxidative stress of nerve cells will not produce ATP in mitochondria. Feet, the energy that maintains normal reaction and activity is insufficient, neuron function can weaken gradually, produce anxious wait for a symptom thereby. Similar results were also found in mouse studies. In this study, the combination of paroxetine and Tianmeng capsule in the treatment of menopausal women with generalized anxiety disorder, played a very good effect, with high safety and effectiveness, worthy of clinical promotion.

\section{References}

[1] Weng Jiehui, Wang Wei, Liu Xinjing, et al. Application effect of fmea in menopausal women with hypertension and the effect on anxiety [J]. Maternal and Child Health Care of China, 2018, 33(5): 1097-1100.

[2] Huo Lei, Zhang Xiuli, Chen Yinfeng, et al. Effects comparison of using sex hormone replacement therapy and routine healthcare in menopausal women health care [J]. China Modern Medicine, 2016, 23(25): 111-113.

[3] Ceng Ying. Clinical value of Kun Tai capsule in the treatment of female menopause insomnia with anxiety and depression [J]. Journal of Practical Gynecologic Endocrinology, 2018, 5(8): 13-14.

[4] Li Zhaosheng. Clinical efficacy of Tianmeng Capsule combined with paroxetine in treatment of menopausal women with generalized anxiety disorder [J]. Chinese Traditional and Herbal Drugs, 2017,48(12): 2498-2501.

[5] Dai Jun, Tan Wei. Observation on the curative effect of paroxetine hydrochloride combined with sweet dream capsuleintreatment of post stroke depression [J]. Chinese Community Doctors, 2016, 32(17): 104+106.

[6] Song Yanyan. Analysis the Cost Effect of Three Therapeutic Schemes in Treatment of Generalized Anxiety Disorder [J]. China Continuing Medical Education, 2016, 8(4): 185-186. 